\title{
Evaluation of a 2-Channel Portable Device and a Predictive Model to Screen for Obstructive Sleep Apnea in a Laboratory Environment
}

\author{
Jianyin Zou, Lili Meng MD, Yupu Liu MSc, Xiaoxi Xu, Suru Liu PhD, Jian Guan PhD, \\ Shankai Yin MD PhD, and Hongliang Yi PhD
}

\begin{abstract}
BACKGROUND: Various portable monitors for identifying obstructive sleep apnea (OSA) have been investigated and reported to enable accurate recording of OSA severity. However, more information is needed from different populations. This study was conducted to evaluate the efficiency of a portable 2-channel sleep apnea device (SleepView) for screening OSA in the Chinese population. METHODS: Ninety-three consecutive subjects underwent simultaneous SleepView testing and laboratory polysomnography (PSG) monitoring. Data were collected and blindly analyzed. The efficiency of the SleepView device and a newly established predictive model for identifying OSA was evaluated in comparison with PSG. RESULTS: Good agreement was evident between the SleepView and PSG based on the apnea-hypopnea index $\left(\mathrm{AHI} ; \mathrm{r}^{2}=0.84, P<.01\right)$. The median AHI yielded by the SleepView was higher than that of PSG: 33.2 (interquartile range of 10.5-53.3) versus 19.2 (interquartile range of 5.2-53.6). The sensitivity and specificity of the SleepView for a PSG AHI of $\geq 5$ were $80.28 \%$ and $95.45 \%$, respectively, and the cutoff was 16.8 . The area under the receiver operating curve for PSG AHIs of $\geq 5,>15$, and $>30$ was $0.923,0.924$, and 0.979 , respectively. When the AHI and oxygen desaturation index calculated by the SleepView were combined with waist circumference, the new predictive model showed a higher sensitivity of $92.96 \%$ and a specificity of $95.45 \%$ for a PSG AHI of $\geq 5$, and the corresponding area under the receiver operating curve was 0.983. CONCLUSIONS: The SleepView device exhibited acceptable diagnostic accuracy for OSA in the Chinese population, especially in the severe OSA group. A practical predictive model comprising waist circumference, AHI, and oxygen desaturation index obtained with the SleepView was highly effective for screening even mild OSA. This simple and practical device may serve as a useful tool to screen for OSA. Further studies are required to validate the diagnostic efficiency of the SleepView in the home environment and in different populations. Key words: obstructive sleep apnea; diagnosis; efficiency; portable monitoring device; predictive model; SleepView; polysomnography. [Respir Care 2015;60(3):356-362. @ 2015 Daedalus Enterprises]
\end{abstract}

\section{Introduction}

Obstructive sleep apnea (OSA) is a common disease characterized by recurrent episodes of apnea and hypop-

\footnotetext{
The authors are affiliated with the Department of Otolaryngology, Affiliated Sixth People's Hospital, Otolaryngology Institute, Shanghai Jiao Tong University, Shanghai, China.
}

The first 2 authors contributed equally to this work.

This study was supported by grant-in-aid SHDC12010209 from the Shanghai Shenkang Hospital Management Center Project of Shanghai. The authors have disclosed no conflicts of interest. nea during sleep. ${ }^{1}$ This is of particular concern because morbidity and mortality associated with untreated OSA have been clearly described in the medical literature. ${ }^{2}$ Epidemiological evidence indicates that undiagnosed OSA is independently associated with increased risks of diabetes mellitus, hypertension, cardiovascular diseases, stroke, day-

\footnotetext{
Correspondence: Hongliang Yi PhD, Department of Otolaryngology, Affiliated Sixth People's Hospital, Otolaryngology Institute, Shanghai Jiao Tong University, 600 Yishan Road, Shanghai 200233, China. E-mail: yihong1@126.com.
}

DOI: $10.4187 /$ respcare. 03626 
time sleepiness, and diminished quality of life. ${ }^{3}$ Prompt diagnosis and treatment of OSA can effectively reduce the risk of developing health consequences and improve general quality of life. However, OSA is often not diagnosed despite improved diagnostic procedures and an increasing awareness of its health consequences. ${ }^{4}$ Nocturnal laboratory-based polysomnography (PSG) is considered timeconsuming, labor-intensive, and costly, although it is the accepted standard for diagnosing OSA. ${ }^{5}$ Potentially alternative diagnostic methods and tools have not been used widely due to their limited efficiency or poor reproducibility. ${ }^{6,7}$ Therefore, development of a simple and practical tool to identify patients with OSA is desirable.

Developments in technology allow small portable monitors to be used for the diagnosis of patients with OSA. Various portable monitors have been investigated. A number of studies have compared these devices, ranging from single-channel recorders to units that record a full polysomnograph, with laboratory PSG. ${ }^{8-10}$ Results suggest that these monitors enable accurate recording of OSA severity. However, the monitors differ markedly in terms of the number and type of signals recorded, the sensors used to record signals, and the electronic processing of the signals. ${ }^{11}$ No unified platform for diagnosis of OSA is available. Thus, as Chesson et al ${ }^{12}$ stated, "results obtained for a particular device are applicable to that device and cannot be extrapolated to other devices, even those of the same class." Additionally, some questions remain, for example, which patients are best suited for portable-monitor testing ${ }^{11}$ ? And what shall we do with the population for whom portable monitors do not work well?

Therefore, we aimed to evaluate the potential value of a portable device (SleepView, BMC Medical, Beijing, China), which allows for the inclusion of 2 high-frequency channels during assessment of cardiorespiratory variables, for screening OSA in the Chinese population and to improve the diagnostic efficiency. We compared the results obtained with this device with those obtained by simultaneous PSG in a sleep laboratory.

\section{Methods}

\section{Subjects and Measurements}

We examined subjects with possible OSA and volunteers with no reported snoring (from the control group of another program) in the sleep center of the Affiliated Sixth People's Hospital of Shanghai Jiao Tong University from May to July 2013. This study was approved by the ethics committee of the Affiliated Sixth People's Hospital. Informed consent was obtained from all subjects. During the sleep center visit, all subjects were asked to complete the Epworth Sleepiness Scale questionnaire and an interna-

\section{QUICK LOOK}

\section{Current knowledge}

Obstructive sleep apnea (OSA) is a common disease, characterized by recurrent episodes of apnea and hypopnea during sleep. Nocturnal laboratory-based polysomnography can be time-consuming, labor-intensive, and costly, although it is the accepted standard for diagnosing OSA. Portable sleep systems for use in the home remain controversial with regard to accuracy.

\section{What this paper contributes to our knowledge}

A portable 2-channel sleep apnea device exhibited acceptable diagnostic accuracy for OSA, especially the severe group. When combined with a practical predictive model comprising waist circumference, apneahypopnea index, and oxygen desaturation index, it was highly effective for screening mild OSA. The 2-channel sleep apnea device might serve as a useful tool to screen for OSA.

tional visual analog scale for snoring intensity before overnight PSG. Body habitus was measured in light clothing and bare feet using standard anthropometric methods. Waist circumference was measured midway between the lower costal margin and the iliac crest, and hip circumference was measured as the maximal girth at the greater trochanter. Neck circumference was measured at the level of the cricothyroid membrane while the subject was standing. These data were recorded as the means of 2 measurements. Body mass index was calculated as weight divided by height squared $\left(\mathrm{kg} / \mathrm{m}^{2}\right)$.

\section{Protocol}

The protocol for this study involved subjects wearing the usual equipment for overnight PSG and the SleepView device. Each recording was evaluated manually by 2 skilled technicians without knowledge of subject identity or the results of the other diagnostic system.

\section{PSG}

The overnight PSG study included acquisition of standard PSG signals, obtained via the Alice 4 PSG system (Philips Respironics, Murrysville, Pennsylvania). The procedure included: an electroencephalogram $(\mathrm{F} 3, \mathrm{~F} 4, \mathrm{C} 1$, C3, O1, O2, M1, M2), a bilateral electroculogram, a submental electromyogram, measurement of oronasal air flow (thermistor and nasal cannula), recording of thoracic and abdominal movements, measurement of $\mathrm{S}_{\mathrm{pO}_{2}}$, recording of 
Table 1. Clinical Characteristics of Subjects

\begin{tabular}{lccc}
\hline \hline \multicolumn{1}{c}{ Clinical Characteristic } & $\begin{array}{c}\text { All Subjects } \\
(N=93)\end{array}$ & $\begin{array}{c}\text { Subjects With AHI }<5 \\
(n=22)\end{array}$ & $\begin{array}{c}\text { Subjects With AHI } \geq 5 \\
(n=71)\end{array}$ \\
\hline Females/males, $n$ & $23 / 70$ & $8 / 14$ & $15 / 56$ \\
Age (mean \pm SD), y & $43.4 \pm 13.2$ & $40.0 \pm 13.9$ & $44.5 \pm 12.9$ \\
BMI (mean \pm SD), $\mathrm{kg} / \mathrm{m}^{2}$ & $26.6 \pm 3.6$ & $24.1 \pm 3.5$ & $27.4 \pm 3.3$ \\
Neck circumference (mean \pm SD), cm & $38.8 \pm 3.5$ & $36.8 \pm 3.0$ & $39.5 \pm 3.4$ \\
Waist circumference (mean \pm SD), cm & $95.4 \pm 10.1$ & $85.4 \pm 9.1$ & $98.5 \pm 8.2$ \\
Hip circumference (mean \pm SD), cm & $100.9 \pm 6.5$ & $95.7 \pm 5.0$ & $102.5 \pm 6.1$ \\
Epworth Sleepiness Scale, median (95\% CI) & $8.0(4.0-12.5)$ & $4.0(0-8.3)$ & $9.0(5.0-14.0)$ \\
Snoring score, median (95\% CI) & $6.0(3.0-8.0)$ & $1.0(0-5.0)$ & $7.0(5.0-9.0)$ \\
& & & \\
AHI = apnea-hypopnea index & & & \\
BMI = body mass index & & & \\
\hline
\end{tabular}

snoring, an electrocardiogram, and recording of leg movements and body position. PSG records were staged manually according to standard criteria by the same technician. Respiratory events were scored according to the American Academy of Sleep Medicine criteria ${ }^{13}$ : apnea was defined as complete cessation of air flow lasting for $\geq 10 \mathrm{~s}$, and hypopnea was defined as either a $\geq 50 \%$ reduction in air flow for $\geq 10$ s or a $<50 \%$ but discernible reduction in air flow accompanied either by a decrease in oxyhemoglobin saturation of $\geq 4 \%$ or by an arousal. The AHI was defined as the number of apnea and hypopnea events/h of sleep time based on the results from overnight PSG.

\section{SleepView Device}

The SleepView device is a 2-channel diagnostic tool designed for screening of sleep-disordered breathing. It consists of an oronasal cannula pressure transducer for air flow and a finger pulse oximetry sensor (measuring $\mathrm{S}_{\mathrm{pO}_{2}}$ and heart rate). It records oral and nasal air flow, snoring, blood oxygen saturation, and pulse. The device uses these recordings to generate a report for screening purposes or to trigger further clinical investigation. This device is like a watch and is held in place by a strap worn around the wrist. The device operates on battery power $(3.0 \mathrm{~V})$. An internal memory of $\sim 18 \mathrm{~h}$ of continuous operation is available. The device was set to continue recording for $7 \mathrm{~h}$, from 10:30 PM to 5:30 AM, as for laboratory PSG. Data from the SleepView device were downloaded and automatically analyzed by SleepView software. The AHI, average $\mathrm{S}_{\mathrm{pO}_{2}}$, minimum $\mathrm{S}_{\mathrm{pO}_{2}}$, percentage of time spent at an $\mathrm{S}_{\mathrm{pO}_{2}}$ of $<90 \%$, and the oxygen desaturation index can be determined using this device. The AHI for the SleepView was defined as the number of events of apnea and hypopnea/h of recording time, instead of sleep time, because no sleep time can be defined by the SleepView.

\section{Statistical Analysis}

Continuous variables are presented as mean $\pm \mathrm{SD}$, with the exception of skewed variables, which are presented as median (with interquartile range). Differences between parameters measured using the 2 tools were examined using the Mann-Whitney $U$ test, the Wilcoxon rank-sum test, or the chi-square test, as appropriate. Correlations between variables were analyzed using Spearman's rank correlation test. Forward conditional logistic regression analysis was performed to establish a diagnostic model, combining parameters from the SleepView. The accuracy of SleepView data and the diagnostic model compared with PSG was examined by constructing receiver operating characteristic curves (ROCs) to identify subjects with undiagnosed OSA. The sensitivity, specificity, positive and negative predictive values, positive and negative likelihood ratios, and overall test accuracy were calculated for both SleepView data alone and the diagnostic model to determine the cutoff values providing maximum diagnostic efficiency.

We considered 2 -sided $P$ values of $<.05$ to indicate statistical significance. Statistical analyses were performed using SPSS 13.0.0 (SPSS, Chicago, Illinois).

\section{Results}

A total of 105 consecutive Chinese subjects participated in the study. Twelve were excluded: 11 with unsuccessful SleepView recordings, inconsecutive signal of air flow, or interruption of oxygen signals, and one who failed to fall asleep. The remaining 93 subjects included 75 subjects with suspected OSA and 18 volunteers with no reported snoring. The proportion of males was $75.3 \%$, the mean age was $43.4 \mathrm{y}$, and the average body mass index as $26.6 \mathrm{~kg} / \mathrm{m}^{2}$. The basic demographics and clinical characteristics of the subjects are shown in Table 1. According to laboratory 
Table 2. Comparison of the Outcomes of Laboratory PSG and the SleepView Device

\begin{tabular}{lccr}
\hline \hline \multicolumn{1}{c}{ Parameter } & PSG & SleepView & \multicolumn{1}{c}{$P$} \\
\hline AHI & $19.2(5.2-53.6)$ & $33.2(10.5-53.3)$ & .005 \\
Hypopnea index & $7.0(2.7-12.0)$ & $1.8(0.8-3.1)$ & $<.001$ \\
Apnea index & $9.2(1.9-39.1)$ & $28.7(8.9-50.2)$ & $<.001$ \\
CT90\% & $2.0(0.2-11.9)$ & $1.3(0.1-6.5)$ & .077 \\
Average $\mathrm{S}_{\mathrm{pO}_{2}}, \%$ & $93.9(92.0-95.0)$ & $95.0(93.5-96.0)$ & $<.001$ \\
Minimum $\mathrm{S}_{\mathrm{pO}_{2}}, \%$ & $82.0(73.5-88.0)$ & $78.0(60.0-84.5)$ & $<.001$ \\
Oxygen desaturation index & $22.4(5.6-49.3)$ & $8.2(2.3-29.7)$ & $<.001$ \\
& & & \\
Values are presented as median (interquartile range). & & \\
PSG = polysomnography & & & \\
AHI = apnea-hypopnea index & & & \\
CT90\% = percentage of time spent with $\mathrm{S}_{\mathrm{pO}_{2}<90 \%}$ & & \\
\hline
\end{tabular}

PSG recordings, the prevalence of an AHI of $\geq 5$ was $76.3 \%$, the prevalence of an $\mathrm{AHI}$ of $>15$ was $58.1 \%$, and the prevalence of an AHI of $>30$ was $41.9 \%$. Most subjects reported frequent snoring $(80.6 \%)$, excessive daytime sleepiness (62.4\%), and witnessed episodes of apnea (78.5\%). By questionnaire assessment, the median Epworth Sleepiness Scale score was 8.0, and the median snoring score was 6.0.

\section{SleepView AHI Versus PSG AHI}

As shown in Table 2, the median AHI yielded by the SleepView was higher than that of PSG, 33.2 (interquartile range of 10.5-53.3) versus 19.2 (interquartile range of 5.2-53.6), and most parameters measured simultaneously by the SleepView and PSG were significantly different $(P<.01)$, with the exception of percentage of time spent at an $\mathrm{S}_{\mathrm{pO}_{2}}$ of $<90 \%(P=.08)$. This outcome showed a lower index of hypopnea and a much higher index of apnea when the SleepView was used. However, the AHI assessed by the SleepView significantly correlated with the AHI simultaneously assessed by PSG $(r=0.92$, $\mathrm{r}^{2}=0.84, P<.01$ ) (Fig. 1A). A Bland-Altman plot of SleepView AHI versus PSG AHI had only 5 (5.4\%) outliers for the SleepView, and most of the AHIs measured by the SleepView fell within 2 SD of the mean PSG value, indicating that differences were reasonably tightly distributed (Fig. 1B). ROC curve analysis was used to evaluate the accuracy of the SleepView in predicting OSA compared with PSG. Figure 2 shows the ROC curves for the AHI from the SleepView versus the AHI from PSG at PSG AHI cutoffs of $\geq 5,>15$, and $>30$. The areas under the ROC curves of the AHIs obtained with the SleepView for the various PSG cutoffs were 0.923, 0.924, and 0.979, respectively, and the corresponding cutoffs of the AHI from the SleepView were 16.8, 22.3, and 37.8, respectively. Table 3 shows the sensitivity, specificity, positive
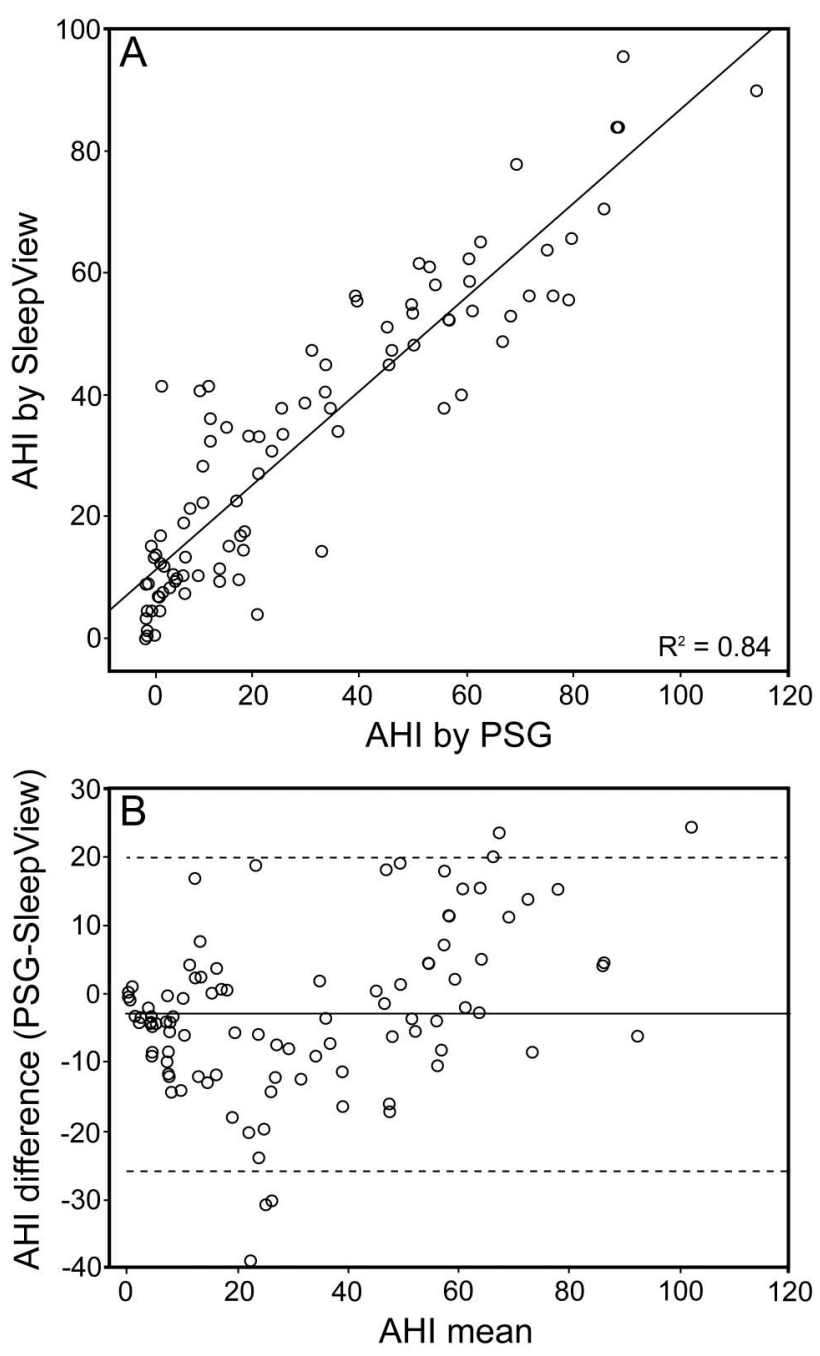

Fig. 1. A: Correlation of the apnea-hypopnea index (AHI) obtained with the SleepView device and simultaneous polysomnography (PSG). B: A Bland-Altman plot of the SleepView AHI versus the PSG AHI measured simultaneously. The dashed lines indicate the upper and lower confidence limits, and the solid line indicates the mean of the differences between the 2 methods of detection.

and negative predictive values, and positive and negative likelihood ratios of the SleepView test compared with PSG at various cutoffs.

\section{Establishment of a Predictive Model}

To improve the efficiency of the SleepView test for identifying patients with mild OSA, we explored prediction models combining clinical parameters and parameters from the SleepView. Using a PSG AHI cutoff of $\geq 5$, all subjects were divided into non-OSA and OSA groups. Next, we included the clinical parameters shown in Table 1 and the SleepView parameters listed in Table 2 in a forward logistic regression analysis. Finally, we generated 


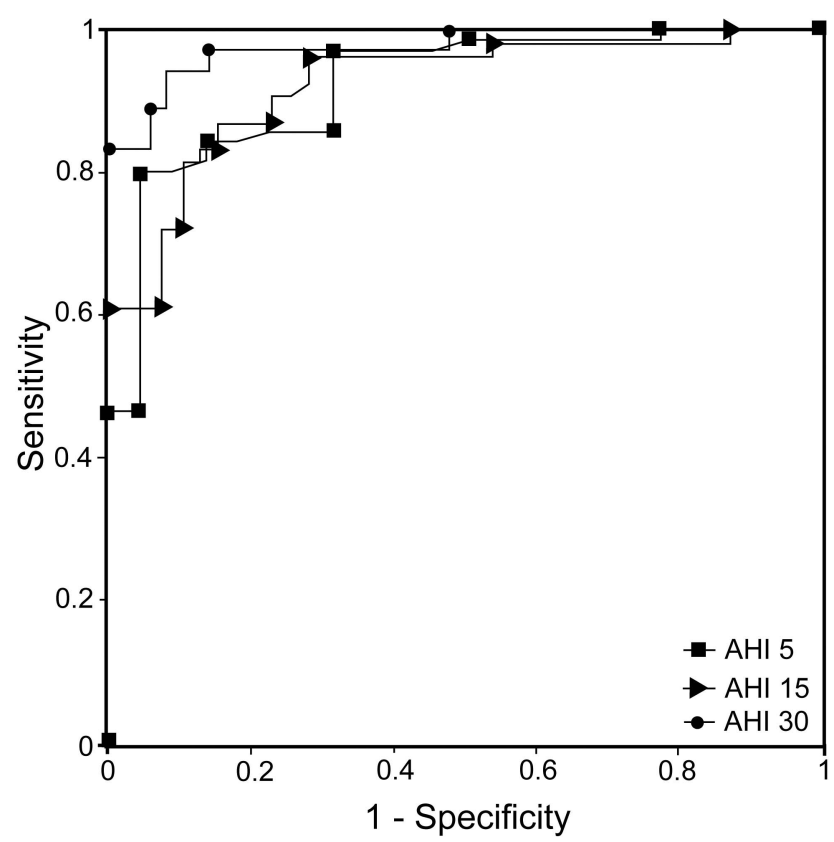

Fig. 2. Receiver operating characteristics curves for the SleepView and polysomnography apnea-hypopnea index (AHI) at various thresholds.

a model: $\quad$ PRE $=1 /[1+\exp (0.114 \times \mathrm{AHI}+0.920 \times$ ODI $+0.235 \times \mathrm{WC}-27.541)]$, where ODI $=$ oxygen desaturation index, and $\mathrm{WC}=$ waist circumference. The area under the ROC curve was 0.983 (95\% CI $0.931-$ 0.999) for identifying OSA with this predictive model (Fig. 3 ). The model showed a sensitivity of $92.96 \%$ (95\% CI 84.33-97.67) and a specificity of $95.45 \%$ (95\% CI 77.1699.88 ) at the optimum cutoff of 0.7346 .

\section{Discussion}

Based on the present work and the standard thresholds commonly used for diagnosis of OSA by laboratory PSG, the SleepView afforded high sensitivity and specificity in identifying OSA, and the performance of the SleepView in

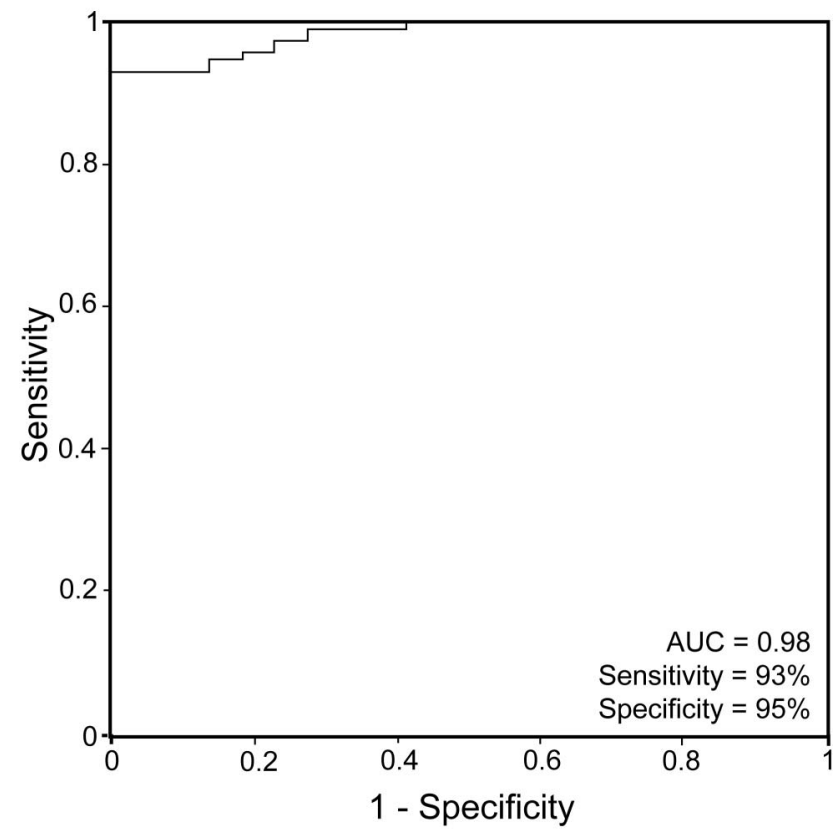

Fig. 3. Receiver operating characteristics curve for the new diagnostic model versus polysomnography at a threshold of the polysomnography apnea-hypopnea index of 5. AUC $=$ area under the curve.

identifying serious OSA was better. When the AHI and oxygen desaturation index obtained with the SleepView were combined with waist circumference, our new diagnostic model exhibited further enhanced accuracy for diagnosis of even mild OSA.

The correlation and Bland-Altman plot analysis demonstrated very good agreement (at almost all levels) between the AHIs calculated by PSG and the SleepView. However, the SleepView AHI was higher than the PSG AHI, with a lower index of hypopnea and a considerably higher index of apnea. This suggests that the SleepView will overestimate the AHI compared with PSG. The SleepView device may have a less sensitive oronasal cannula air-flow pressure transducer, which may identify hypopnea as an apnea. This may be the reason why the SleepView AHI was

Table 3. Efficiency of OSA Identification by SleepView AHI Versus PSG AHI Using Various Thresholds

\begin{tabular}{|c|c|c|c|c|c|c|c|c|}
\hline $\begin{array}{l}\text { PSG } \\
\text { AHI }\end{array}$ & $\begin{array}{l}\text { Sensitivity, } \\
\%(95 \% \text { CI }\end{array}$ & $\begin{array}{l}\text { Specificity, } \\
\%(95 \% \text { CI }\end{array}$ & $\begin{array}{c}\text { Positive } \\
\text { predictive value, } \\
\%(95 \% \mathrm{CI})\end{array}$ & $\begin{array}{c}\text { Negative } \\
\text { predictive value, } \\
\%(95 \% \mathrm{CI})\end{array}$ & $\begin{array}{l}\text { Positive } \\
\text { likelihood ratio } \\
(95 \% \mathrm{CI})\end{array}$ & $\begin{array}{l}\text { Negative } \\
\text { likelihood ratio } \\
(95 \% \mathrm{CI})\end{array}$ & $\begin{array}{c}\text { ROC } \\
(95 \% \mathrm{CI})\end{array}$ & Cutoff \\
\hline
\end{tabular}

$\begin{array}{lllllllll}\geq & 80.28(69.13-88.78) & 95.45(77.16-99.88) & 98.28(90.76-99.96) & 60.00(42.11-76.13) & 17.66(2.59-120.28) & 0.21(0.13-0.33) & 0.923(0.849-0.968) & \geq 16.8\end{array}$

$\begin{array}{lllllllll}>15 & 87.04(75.10-94.63) & 84.62(69.47-94.14) & 88.68(76.97-95.73) & 82.50(67.22-92.66) & 5.66(2.69-11.90) & 0.15(0.08-0.31) & 0.924(0.850-0.969) & >22.3\end{array}$

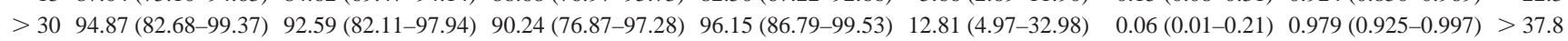

ROC $=$ receiver operating characteristic 
formed by a lower index of hypopnea and a higher index of apnea compared with PSG. On ROC curve analysis, the SleepView measurement had the highest specificity and sensitivity at an AHI of $>16.8$ when used to identify OSA defined by PSG as an AHI of $\geq 5$. Similarly, the SleepView with an AHI of $>22.3$ afforded the greatest accuracy in terms of identifying moderate and severe OSA defined by a PSG AHI of $>15$, and a SleepView AHI of $>37.8$ afforded the greatest accuracy in identifying severe OSA defined by a PSG AHI of $>30$. The SleepView measurement performed better in terms of identifying serious OSA compared with mild or moderate OSA. This was in line with previous reports. ${ }^{11,14}$

In a published guideline for portable monitoring, the American Academy of Sleep Medicine recommended that portable monitoring "may be used as an alternative to PSG for the diagnosis of OSA in patients with a high pretest probability of moderate to severe OSA." 15 However, by combining parameters obtained from the SleepView test with other clinical parameters, we developed a new diagnostic model with a high sensitivity of $92.96 \%$ and a specificity of $95.45 \%$ for identifying OSA defined by a PSG AHI of $\geq 5$; therefore, the model may be satisfactory for diagnosis of even mild OSA.

An accurate and effective portable device might overcome the problems associated with PSG, which is the accepted standard for diagnosis of OSA. PSG is timeconsuming and costly and cannot be used to evaluate large numbers of patients suspected to have OSA because sleep centers are rare, particularly in undeveloped regions. Undiagnosed OSA and associated complications increase health-care costs. In contrast, a portable device could reduce the price and effort of OSA diagnosis and might reduce patients' discomfort, with fewer wires compared with PSG. Additionally, in recent years, there has been a dramatic shift from PSG to home sleep testing for OSA to reduce health-care costs associated with OSA by facilitating diagnosis and enabling more timely therapeutic intervention. Hence, our findings may be potentially helpful for clinical practice.

Several potential limitations of this study should be considered. First, data loss was $11.4 \%$ (12 of 105 subjects) principally because the portable device was left unattended throughout the night. Another reason may be discomfort caused by simultaneous use of 2 instruments, especially the 2 oronasal cannulas used to monitor air flow. As mentioned in other studies, portable monitor recordings are more likely to suffer a $4-33 \%$ data loss, which is more than laboratory PSG. ${ }^{16,17}$ Second, we did not perform the SleepView test in the home. The location of the study (a sleep laboratory vs the home) may have affected the results, although no significant differences between laboratory and home tests in terms of diagnosis of moderate and severe OSA have been noted. ${ }^{14,18}$ Third, the AHI calcu- lated by the SleepView device was based on recording time, not sleep time, which had an effect on the value. Additionally, all subjects recruited from our sleep center were Chinese and were suspected to have OSA. Therefore, the outcomes might be different in the general population and with different races. The use of the SleepView monitor in the home and with different populations should be further investigated. Fourth, as a 2-channel device without chest-wall breathing movement signals, the SleepView is unable to distinguish between central and obstructive apneas; this may limit the use of the SleepView in certain studies that require detailed respiratory events.

\section{Conclusions}

In this study, the SleepView device afforded acceptable diagnostic accuracy for OSA. The predictive model, comprising waist circumference, AHI, and oxygen desaturation index obtained with the SleepView, enabled highly effective diagnosis of mild OSA. Our results suggest that the device could be a complementary tool for screening OSA. Further studies are required to validate the diagnostic efficiency of the SleepView in a home environment and in the general population.

\section{ACKNOWLEDGMENTS}

We thank Mr Jin You, Mr Yin Li, and Mr Qiang Song (Department of Otolaryngology, Affiliated Sixth People's Hospital, Otolaryngology Institute, Shanghai Jiao Tong University, Shanghai, China) for their assistance in recruitment of subjects and data collection.

\section{REFERENCES}

1. Berg S. Obstructive sleep apnoea syndrome: current status. Clin Respir J 2008;2(4):197-201.

2. Marin JM, Carrizo SJ, Vicente E, Agusti AG. Long-term cardiovascular outcomes in men with obstructive sleep apnoea-hypopnoea with or without treatment with continuous positive airway pressure: an observational study. Lancet 2005;365(9464):1046-1053.

3. Young T, Peppard PE, Gottlieb DJ. Epidemiology of obstructive sleep apnea: a population health perspective. Am J Respir Crit Care Med 2002;165(9):1217-1239.

4. Simpson L, Hillman DR, Cooper MN, Ward KL, Hunter M, Cullen $S$, et al. High prevalence of undiagnosed obstructive sleep apnoea in the general population and methods for screening for representative controls. Sleep Breath 2013;17(3):967-973.

5. American Academy of Sleep Medicine Task Force. Sleep-related breathing disorders in adults: recommendations for syndrome definition and measurement techniques in clinical research. Sleep 1999; 22(5):667-689.

6. Silva GE, Vana KD, Goodwin JL, Sherrill DL, Quan SF. Identification of patients with sleep disordered breathing: comparing the four-variable screening tool, STOP, STOP-Bang, and Epworth Sleepiness Scales. J Clin Sleep Med 2011;7(5):467-472.

7. Aaronson JA, van Bezeij T, van den Aardweg JG, van Bennekom CA, Hofman WF. Diagnostic accuracy of nocturnal oximetry for detection of sleep apnea syndrome in stroke rehabilitation. Stroke 2012;43(9):2491-2493. 


\section{Portable Device for OSA ScReEning}

8. Crowley KE, Rajaratnam SM, Shea SA, Epstein LJ, Czeisler CA, Lockley SW. Evaluation of a single-channel nasal pressure device to assess obstructive sleep apnea risk in laboratory and home environments. J Clin Sleep Med 2013;9(2):109-116.

9. Ferré A, Sampol G, Jurado MJ, Cambrodi R, Lloberes P, Romero O. Neurophysiological two-channel polysomnographic device in the diagnosis of sleep apnea. J Clin Sleep Med 2012;8(2): 163-168.

10. Driver HS, Pereira EJ, Bjerring K, Toop F, Stewart SC, Munt PW, Fitzpatrick MF. Validation of the MediByte type 3 portable monitor compared with polysomnography for screening of obstructive sleep apnea. Can Respir J 2011;18(3):137-143.

11. Kuna ST. Portable-monitor testing: an alternative strategy for managing patients with obstructive sleep apnea. Respir Care 2010;55(9): 1196-1215.

12. Chesson AL Jr, Berry RB, Pack A. Practice parameters for the use of portable monitoring devices in the investigation of suspected obstructive sleep apnea in adults. Sleep 2003;26(7):907-913.

13. Iber C, Ancoli-Israel S, Chesson AL Jr, Quan SF. For the American Academy of Sleep Medicine. The AASM Manual for the scoring of sleep and associated events: rules, terminology and technical specifica- tions. Westchester, Illinois: American Academy of Sleep Medicine; 2007.

14. Oktay B, Rice TB, Atwood CW Jr, Passero M Jr, Gupta N, Givelber R, et al. Evaluation of a single-channel portable monitor for the diagnosis of obstructive sleep apnea. J Clin Sleep Med 2011;7(4):384-390.

15. Collop NA, Anderson WM, Boehlecke B, Claman D, Goldberg R, Gottlieb DJ, et al. Clinical guidelines for the use of unattended portable monitors in the diagnosis of obstructive sleep apnea in adult patients. J Clin Sleep Med 2007;3(7):737-747.

16. Redline S, Tosteson T, Boucher MA, Millman RP. Measurement of sleep-related breathing disturbances in epidemiologic studies. Assessment of the validity and reproducibility of a portable monitoring device. Chest 1991;100(5):1281-1286

17. Gagnadoux F, Pelletier-Fleury N, Philippe C, Rakotonanahary D, Fleury B. Home unattended vs hospital telemonitored polysomnography in suspected obstructive sleep apnea syndrome: a randomized crossover trial. Chest 2002;121(3):753-758.

18. Erman MK, Stewart D, Einhorn D, Gordon N, Casal E. Validation of the ApneaLink for the screening of sleep apnea: a novel and simple single-channel recording device. J Clin Sleep Med 2007;3(4): 387-392. 